TAIWANESE JOURNAL OF MATHEMATICS

Vol. 12, No. 9, pp. 2405-2425, December 2008

This paper is available online at http://www.tjm.nsysu.edu.tw/

\title{
SOME FURTHER RESULTS ON ENTIRE FUNCTIONS SHARING A POLYNOMIAL WITH THEIR LINEAR DIFFERENTIAL POLYNOMIALS
}

\author{
Xiao-Min Li and Hong-Xun Yi
}

\begin{abstract}
In this paper, we study the growth of all solutions of a linear differential equation. From this we obtain some uniqueness theorems of a nonconstant entire function and its linear differential polynomials having the same fixed points. The results in this paper also improve some known results. Two example are provided to show that the results in this paper are best possible.
\end{abstract}

\section{Introduction ANd Main Results}

In this paper, by meromorphic functions we will always mean meromorphic functions in the complex plane. We adopt the standard notations in the Nevanlinna theory of meromorphic functions as explained in [5],[7],[9]. It will be convenient to let $E$ denote any set of positive real numbers of finite linear measure, not necessarily the same at each occurrence. For any nonconstant meromorphic function $h(z)$, we denote by $S(r, h)$ any quantity satisfying

$$
S(r, h)=o(T(r, h)) \quad(r \rightarrow \infty, r \notin E) .
$$

Let $h$ be a nonconstant meromorphic function, and $k$ a positive integer. We use $\bar{N}_{k)}(r, h)$ to denote the reduced counting function of poles of $h$, whose multiplicities are not greater than $k$. Likewise, we use $\bar{N}_{(k}(r, h)$ to denote the reduced counting function of poles of $h$, whose multiplicities are not less than $k$. When multiplicities are duly counted in the above notations, we use $N_{k)}(r, h)$ and $N_{(k}(r, h)$ to indicate them (see [11]).

Received January 17, 2007, Accepted June 9, 2007.

Communicated by Der-Chen Chang.

2000 Mathematics Subject Classification: 30D35, 30D20.

Key words and phrases: Entire function, Order of growth, Shared value, Uniqueness.

Project supported by the NSFC (No. A0324617), the RFDP (No. 20060422049) and the NSFC (No. 10771121). 
Let $f$ and $g$ be two nonconstant meromorphic functions, and let $P$ be a polynomial. We say that $f$ and $g$ share $P \mathrm{CM}$, provided that $f-P$ and $g-P$ have the same zeros with the same multiplicities. Similarly, we say that $f$ and $g$ share $P$ IM, provided that $f-P$ and $g-P$ have the same zeros ignoring multiplicities (see[11]). In this paper, we also need the following two definitions.

Definition 1.1. Let $f$ be a nonconstant entire function, the order of $f$, denoted $\sigma(f)$, is defined by

$$
\sigma(f)=\limsup _{r \rightarrow \infty} \frac{\log T(r, f)}{\log r}=\limsup _{r \rightarrow \infty} \frac{\log \log M(r, f)}{\log r},
$$

where and in what follows, $M(r, f)=\max _{|z|=r}\{|f(z)|\}$.

Definition 1.2. Let $f$ be a nonconstant meromorphic function, the hyper-order of $f$, denoted $\sigma_{2}(f)$, is defined by

$$
\sigma_{2}(f)=\limsup _{r \rightarrow \infty} \frac{\log \log T(r, f)}{\log r}==\limsup _{r \rightarrow \infty} \frac{\log \log \log M(r, f)}{\log r} .
$$

In 1976, L. A. Rubel and C. C. Yang proved the following theorem.

Theorem A. (see [8]). Let $f$ be a nonconstant entire function. If $f$ and $f^{\prime}$ share two finite distinct values $C M$, then $f \equiv f^{\prime}$.

In 1996, R.Brück proved the following theorems.

Theorem B. (see [1]). Let $f$ be a nonconstant entire function satisfying $\sigma_{2}(f)<\infty$, and $\sigma_{2}(f)$ is not a positive integer. If $f$ and $f^{\prime}$ share the value $0 C M$, then $f \equiv c f^{\prime}$ for some constant $c \neq 0$.

Theorem C. (see [1]). Let $f$ be a nonconstant entire function. If $f$ and $f^{\prime}$ share $1 C M$, and if $N\left(r, \frac{1}{f^{\prime}}\right)=S(r, f)$, then $f-1 \equiv c\left(f^{\prime}-1\right)$ for some constant $c \neq 0$.

In the same paper, Brück made the following conjecture.

Conjecture 1.1. (see [1]). Let $f$ be a nonconstant entire function satisfying $\sigma_{2}(f)<\infty$, and $\sigma_{2}(f)$ is not a positive integer. If $f$ and $f^{\prime}$ share one finite value $a \mathrm{CM}$, then $f-a \equiv c\left(f^{\prime}-a\right)$ for some constant $c \neq 0$.

Consider the differential equation

$$
f^{\prime}-e^{Q(z)} f=1
$$

where $Q(z)$ is an entire function. 
In 1998, G. G. Gundersen and L. Z. Yang proved that the conjecture is true for $a \neq 0$, provided that $f$ satisfies the additional assumption $\sigma(f)<\infty$. In fact, they proved the following results.

Theorem D. (see [4, Lemma 1]). Let $Q(z)$ be a nonconstant polynomial. Then every solution of (1.1) is an entire function of infinite order.

Theorem E. (see [4, Theorem 1]). Let $f$ be a nonconstant entire function of finite order. If $f$ and $f^{\prime}$ share one finite value a $C M$, then $f-a \equiv c\left(f^{\prime}-a\right)$ for some constant $c \neq 0$.

Let

$$
L[f]=f^{(k)}+a_{k-1} f^{(k-1)}+\cdots+a_{1} f^{\prime}+a_{0} f,
$$

where $k$ is a positive integer, and $a_{0}, a_{1}, \cdots a_{k-1}, a_{k}$ are $k$ finite complex numbers.

In this paper, we will prove the following results, which improve Theorem D and Theorem E.

Theorem 1.1. Let $P(z)$ and $Q_{j}(z)(j=1,2)$ be polynomials. If $f$ is a nonconstant solution of the equation

$$
L[f]-Q_{1}=\left(f-Q_{2}\right) \cdot e^{P(z)},
$$

where $L[f]$ is defined by (1.2), then one of the following three cases will occur.

(i) If $f$ is a polynomial or $P(z)$ is a constant, then $L[f]-Q_{1}=c\left(f-Q_{2}\right)$, where $c$ is a finite nonzero complex number;

(ii) If $P(z)$ is not a constant and $\mu(f)>1$, then $\sigma(f)=\infty$ and $\sigma_{2}(f)=\gamma_{P}$, where and in what follows, $\mu(f)$ denotes the lower order of $f, \gamma_{P}$ denotes the degree of $P(z)$;

(iii) If $P(z)$ is not a constant and $\mu(f) \leq 1$, then $\mu(f)=1$ and $P(z)=$ $p_{1} z+p_{0}$, where $p_{1}(\neq=0)$ and $p_{0}$ are two finite complex numbers, moreover, $a_{0}, a_{1}, \cdots a_{k-2}$ and $a_{k-1}(k \geq 2)$ are not all equal to zero.

From Theorem 1.1 we get the following three corollaries, of which Corollary 1.1 improves Theorem D, Corollary 1.2 improves Theorem E.

Corollary 1.1. Let $P(z)$ be a nonconstant polynomial such that $\gamma_{p} \geq 2$. Then every solution of (1.3) is an entire function of infinite order, and $\sigma_{2}(f)=\gamma_{P}$, where $\gamma_{P}$ is the degree of $P(z)$.

Corollary 1.2. Let $f$ be a nonconstant entire function of finite order, and let $Q_{j}(z)(j=1,2)$ be two polynomials. If $f-Q_{2}$ and $L[f]-Q_{1}$ share $0 C M$, then $\mu(f)=\sigma(f)=1$ and one of the following two cases will occur. 
(i) $L[f]-Q_{1}=c\left(f-Q_{2}\right)$, where $c$ is a finite nonzero complex number;

(ii) $L[f]-Q_{1}=\left(f-Q_{2}\right) \cdot e^{p_{1} z+p_{0}}$, where $p_{1}(\neq=0)$ and $p_{0}$ are two finite complex numbers, $a_{0}, a_{1}, \cdots a_{k-2}$ and $a_{k-1}(k \geq 2)$ are not all equal to zero.

Corollary 1.3. Let $P(z)$ and $Q_{j}(z)(j=1,2)$ be polynomials. If $f$ is a solution of (1.3) such that $\sigma_{2}(f)$ is not a positive integer, then the conclusions (i) and (ii) of Corollary 1.2 hold.

Proceeding as in the proof of Theorem 1.1 in Section 3 of this paper, we get the following theorem.

Theorem 1.2. Let $P(z)$ and $Q_{j}(z)(j=1,2)$ be polynomials. If $f$ is a nonconstant solution of the equation $f^{(k)}-Q_{1}=\left(f-Q_{2}\right) \cdot e^{P(z)}$, where $k(\geq 1)$ is a positive integer, then $\sigma_{2}(f)=\gamma_{p}$.

Example 1.1. Let $f$ be a solution of the differential equation

$$
f^{\prime}-z=(f-z) \cdot e^{z^{n}},
$$

where $n$ is a positive integer. Since (1.4) can be rewritten by

$$
f^{\prime}-f \cdot e^{z^{n}}=z\left(1-e^{z^{n}}\right),
$$

from (1.5) and Lemma 2.3 in Section 2 of this paper we can see that every solution of (1.5) is a nonconstant entire function. Moreover, it follows from (1.4) that $f-z$ and $f^{\prime}-z$ share $0 \mathrm{CM}$. From Lemma 1.1.2 in [7] and in the same manner as in the proof of (3.16) in the proof of Theorem 1.1 in Section 3 of this paper, we get $\mu_{2}(f)=n$, where and in what follows, $\mu_{2}(f)$ denotes the lower hyper order of $f$. This example shows that the condition " $a_{0}, a_{1}, \ldots a_{k-2}$ and $a_{k-1}(k \geq 2)$ are not all equal to zero" in (iii) of Theorem 1.1 and (ii) of Corollaries 1.2-1.3 is best possible.

Example 1.2. Let $f=\left(e^{z}-1\right)^{2}$ and $L[f]=f^{(3)}-3 f^{\prime \prime}+\frac{5}{3} f^{\prime}-f$. Then we verify that $\mu(f)=\sigma(f)=1$ and $L[f]-1=(f-1) \cdot e^{-z}$. This example shows that the conclusion (iii) of Theorem 1.1 and (ii) of Corollaries 1.2-1.3 can occur.

Corollary 1.4. Let $P(z)$ be a polynomial such that $\gamma_{P} \neq 1$, and let $a(\neq 0)$ be a finite complex number. Suppose that $f$ is a nonconstant solution of the differential equation

$$
\frac{L[f]-z}{f-z}=e^{P(z)}
$$

where $L[f]$ is defined as in (1.2), and that $\sigma_{2}(f)$ is not a positive integer. If $f$ and $L[f]$ share the value a $I M$, then $f \equiv L[f]$. 
Proof of Corollary 1.4. First, from Corollary 1.3 and the condition $\gamma_{P} \neq 1$ we get $L[f]-z=c(f-z)$. On the other hand, from the condition that $f$ and $L[f]$ share a IM and Milloux $<$ s inequality (see [5, Theorem 3.2]) we see that there exists one point $z_{0}$ such that $L[f]\left(z_{0}\right)=f\left(z_{0}\right)=a \neq z_{0}$. From this and $L[f]-z=c(f-z)$ we get the conclusion of Corollary 1.4.

Corollary 1.5. Let $P(z)$ be a polynomial, such that $\gamma_{P} \neq 1$, and $a_{0}$ be a constant. Suppose that $f$ is a nonconstant solution of the differential equation (1.6), such that $\sigma_{2}(f)$ is not a positive integer, where

$$
L[f]=f^{\prime}+a_{0} f .
$$

If $f$ and $L[f]$ share $0 I M$, then $f \equiv L[f]$.

Proof. First, from Corollary 1.3 and the assumptions of Corollary 1.5 we get

$$
\frac{f^{\prime}(z)+a_{0} f(z)-z}{f(z)-z} \equiv c,
$$

where $c$ is a nonzero constant. If $c=1$, then from (1.8) we can get the conclusion of Corollary 1.5. Next we assume that $c \neq 1$. Since (1.8) can be rewritten as

$$
f^{\prime}+\left(a_{0}-c\right) f=(1-c) z,
$$

which is a linear $O D E$ of order 1. Suppose that there exists a finite complex number $z_{0}$ such that $f\left(z_{0}\right)=0$, then from the condition that $f$ and $L[f]$ share 0 IM we have $L[f]\left(z_{0}\right)=f^{\prime}\left(z_{0}\right)+a_{0} f\left(z_{0}\right)=0$, and so $f^{\prime}\left(z_{0}\right)=0$. Combining (1.9) we deduce $z_{0}=0$. That is, $f$ and $L[f]$ have at most one zero $z=0$. We discuss the following two cases.

Case 1. Suppose that $a_{0}=c$. Then from (1.9) and the condition that $f$ has at most one zero $z=0$, we deduce

$$
\begin{aligned}
& f(z)=\frac{1}{2}(1-c) z^{2} \\
& L[f]=f^{\prime}+c f=\frac{1}{2} c(1-c) z^{2}+(1-c) z .
\end{aligned}
$$

Noting that $c \neq 1$ and that $L[f]$ has at most one zero $z=0$, from (1.10) we get a contradiction.

Case 2. uppose that $a_{0} \neq c$. Then the general solution (1.9) is

$$
f=c_{1} e^{\left(c-a_{0}\right) z}+\frac{(1-c) z}{a_{0}-c}+\frac{c-1}{\left(a_{0}-c\right)^{2}},
$$


where $c_{1}$ is a finite complex number. Noting that $c \neq 1$ and that $f$ has at most one zero $z=0$, from (1.11) we get a contradiction.

In 1995, H. X. Yi and C. C. Yang posed the following question.

Question 1.1. (see [11, pp. 398]). Let $f$ be a nonconstant meromorphic function, and let $a$ be a nonzero constant. If $f, f^{(n)}$ and $f^{(m)}$ share the value $a$ $\mathrm{CM}$, where $n$ and $m(n<m)$ are distinct positive integers not all even or odd, then can we get the result $f \equiv f^{(n)}$ ?

Regarding Question 1.1, G. G. Gundersen and L. Z. Yang proved the following result in 1998.

Theorem F. (see [4, Theorem 2]). Let $f$ be a nonconstant entire function of finite order, let a be a nonzero constant, and let $n$ be a positive integer. If the value $a$ is shared by $f, f^{(n)}$ and $f^{(n+1)} I M$, and shared by $f^{(n)}$ and $f^{(n+1)} C M$, then $f \equiv f^{\prime}$.

In this paper, we will prove the following result, which supplements Theorem F.

Theorem 1.3. Let $f$ be a nonconstant solution of the differential equation

$$
\frac{L^{\prime}[f]-z}{L[f]-z}=e^{P}
$$

where $L[f]$ is defined as in (1.7), and $P(z)$ is a polynomial. If $\sigma_{2}(f)$ is not a positive integer, and if $f(z)$ and $L[f]$ share $z I M$, then $e^{P}$ is a constant, and $f$ is given by one of the following two expressions.

(i) $f=c_{1} z+a_{0} c_{1}\left(1-c_{1}\right)$ and $a_{0}^{2}-a_{0}+1=0$, where $c_{1}\left(\neq 0,1,1 / a_{0}\right)$ is a finite complex number, and $e^{P} \equiv 1 /\left(1-a_{0} c_{1}\right)$.

(ii) $f=d_{1} e^{z}$ and $a_{0}=0$, where $d_{1}(\neq 0)$ is a finite complex constant, and $e^{P} \equiv 1$.

\section{Some Lemmas}

Lemma 2.1. (see [6, pp36-37] or [7, Theorem 3.1]). If $f$ is an entire function of order $\sigma(f)$, then

$$
\sigma(f)=\limsup _{r \rightarrow \infty} \frac{\log \nu(r, f)}{\log r}
$$

where, and in the sequel, $\nu(r, f)$ denotes the central-index of $f(z)$.

Lemma 2.2. (see [2, Lemma 2] or [3, Lemma 4]). If $f$ is a transcendental entire function of hyper-order $\sigma_{2}(f)$, then

$$
\sigma_{2}(f)=\limsup _{r \rightarrow \infty} \frac{\log \log \nu(r, f)}{\log r} .
$$


Lemma 2.3. (see[7, Proposition 8.1]). Let

$$
f^{(n)}+a_{n-1} f^{(n-1)}+\cdots+a_{1} f^{\prime}+a_{0} f=F(z),
$$

where $a_{0}(\not \equiv 0), a_{1}, \cdots, a_{n-1}$ and $F(\not \equiv 0)$ are entire functions. Then all solutions of (2.1) are entire functions.

Lemma 2.4. (see [11, Theorem 1.49]). Suppose that $f_{1}, f_{2}, \cdots, f_{n}$ are linearly independent meromorphic functions satisfying the following identity

$$
\sum_{i=1}^{n} f_{i} \equiv 1 .
$$

If

$$
\sum_{i=1}^{n} \bar{N}\left(r, f_{i}\right)=S(r)
$$

where

$$
S(r)=o(T(r))(r \rightarrow \infty, r \notin E), T(r)=\max _{1 \leq i \leq n}\left\{T\left(r, f_{i}\right)\right\},
$$

then

$$
T(r) \leq \sum_{i=1}^{n} N\left(r, \frac{1}{f_{i}}\right)+S(r) .
$$

Lemma 2.5. Let $f_{j}(j=1,2, \cdots, n)$ be nonconstant meromorphic functions satisfying

$$
N\left(r, \frac{1}{f_{j}}\right)+\bar{N}\left(r, f_{j}\right)=S\left(r, f_{j}\right) \quad(j=1,2, \cdots, n),
$$

and let

$$
F \equiv a+\sum_{j=1}^{n} f_{j}
$$

where $a$ is a meromorphic function satisfying $a \not \equiv 0$. If $F$ is not constant, and $T(r, a)=S(r, F)$, then

$$
T(r, F)=N\left(r, \frac{1}{F}\right)+S(r, F) .
$$

Proof. Obviously, $\sum_{j=1}^{n} f_{j} \not \equiv 0$. Without loss of generality, let

$$
\sum_{j=1}^{n} f_{j} \equiv \sum_{j=1}^{k} c_{j} f_{j}
$$


where $f_{1}, f_{2}, \cdots, f_{k}$ are linearly independent, and $c_{1}, c_{2}, \cdots, c_{k}$ are nonzero constants. Let

$$
g_{j}=-\frac{c_{j} f_{j}}{a}(j=1,2, \cdots, k) \quad \text { and } \quad g_{k+1}=\frac{F}{a} .
$$

From (2.3), (2.5) and (2.6) we have

$$
\sum_{j=1}^{k+1} g_{j} \equiv 1
$$

It is easy to see that $g_{1}, g_{2}, \cdots, g_{k+1}$ are linearly independent. By Lemma 2.4, (2.2), (2.3), (2.6) and (2.7) we obtain

$$
T(r) \leq \sum_{i=1}^{k+1} N\left(r, \frac{1}{f_{i}}\right)+S(r) \leq N\left(r, \frac{1}{F}\right)+S(r) \leq T(r, F)+S(r),
$$

where

$$
T(r)=\max _{1 \leq j \leq k+1}\left\{T\left(r, g_{j}\right)\right\} \quad \text { and } \quad S(r)=o(T(r))(r \rightarrow \infty, r \notin E) .
$$

From (2.8) we can obtain (2.4).

Lemma 2.6. (see [11, Theorem 1.57]). Suppose that $f_{1}, f_{2}, f_{3}$ are meromorphic functions satisfying

$$
f_{1}+f_{2}+f_{3} \equiv 1
$$

If $f_{1}$ is not a constant and

$$
\sum_{i=1}^{3} N\left(r, \frac{1}{f_{i}}\right)+2 \sum_{i=1}^{3} \bar{N}\left(r, f_{i}\right)<\lambda T\left(r, f_{1}\right)+S\left(r, f_{1}\right),
$$

where $\lambda<1$, then $f_{2} \equiv 1$ or $f_{3} \equiv 1$.

Lemma 2.7. Suppose that $\alpha$ and $\beta$ are nonconstant entire functions, and that $a_{1}, a_{2}, b_{1}$ and $b_{2}$ are meromorphic functions satisfying $T\left(r, a_{1}\right)+T\left(r, a_{2}\right)=$ $S\left(r, e^{\alpha}\right), T\left(r, b_{1}\right)+T\left(r, b_{2}\right)=S\left(r, e^{\beta}\right)$ and $a_{1} a_{2} b_{1} b_{2} \not \equiv 0$. If $a_{1} e^{\alpha}-a_{2}$ and $b_{1} e^{\beta}-b_{2}$ share $0 I M$, then one of the following relations holds:

(i) $a_{1} b_{2} e^{\alpha} \equiv a_{2} b_{1} e^{\beta}$

(ii) $a_{1} b_{1} e^{\alpha+\beta} \equiv a_{2} b_{2}$ 
Proof. By the second fundamental theorem, we have

$$
T\left(r, e^{\alpha}\right)=\bar{N}\left(r, \frac{1}{a_{1} e^{\alpha}-a_{2}}\right)+S\left(r, e^{\alpha}\right)=N_{1)}\left(r, \frac{1}{a_{1} e^{\alpha}-a_{2}}\right)+S\left(r, e^{\alpha}\right)
$$

and

$$
T\left(r, e^{\beta}\right)=\bar{N}\left(r, \frac{1}{b_{1} e^{\beta}-b_{2}}\right)+S\left(r, e^{\beta}\right)=N_{1)}\left(r, \frac{1}{b_{1} e^{\beta}-b_{2}}\right)+S\left(r, e^{\beta}\right)
$$

Let

$$
H=\frac{a_{1} e^{\alpha}-a_{2}}{b_{1} e^{\beta}-b_{2}} .
$$

Noting that $a_{1} e^{\alpha}-a_{2}$ and $b_{1} e^{\beta}-b_{2}$ share $0 \mathrm{IM}$, from (2.9)-(2.11) we obtain

$$
N(r, H)=S\left(r, e^{\alpha}\right) \quad \text { and } \quad N\left(r, \frac{1}{H}\right)=S\left(r, e^{\alpha}\right) .
$$

By (2.11) we get

$$
\frac{a_{1}}{a_{2}} e^{\alpha}-\frac{b_{1}}{a_{2}} H e^{\beta}+\frac{b_{2}}{a_{2}} H=1 .
$$

From (2.12) and (2.13), by Lemma 2.6 we obtain $\frac{b_{2}}{a_{2}} H=1$ or $-\frac{b_{2}}{a_{2}} H e^{\beta}=1$.

If $\frac{b_{2}}{a_{2}} H=1$, from (2.13) we have $\frac{a_{1}}{a_{2}} e^{\alpha}=\frac{b_{1}}{a_{2}} H e^{\beta}$. From this we have the relation (i) of Lemma 2.7.

If $-\frac{b_{1}}{a_{2}} H e^{\beta}=1$, from (2.13) we have $\frac{a_{1}}{a_{2}} e^{\alpha}=-\frac{b_{2}}{a_{2}} H$. From this we have the relation (ii) of Lemma 2.7.

Lemma 2.8. Suppose that $R_{1}$ and $R_{2}$ are rational functions, and that $a_{1}$ and $a_{2}$ are two constants satisfying $0<\left|a_{1}\right| \leq\left|a_{2}\right|$ and $a_{1} \neq a_{2}$. Then there exists $a$ constant $A(>1)$ such that

$$
A T\left(r, e^{a_{1} z}\right) \leq T\left(r, R_{1} e^{a_{1} z}+R_{2} e^{a_{2} z}\right)+O(\log r) .
$$

Proof. It is easy to see that

$$
T\left(r, e^{a_{1} z}\right)=\frac{\left|a_{1}\right| r}{\pi}, \quad T\left(r, e^{a_{2} z}\right)=\frac{\left|a_{2}\right| r}{\pi} .
$$

Let $a_{j}=\left|a_{j}\right| e^{i \theta_{j}}(j=1,2)$, where $0 \leq \theta_{j}<2 \pi(j=1,2)$. We have

$$
\begin{aligned}
& T\left(r, R_{1} e^{a_{1} z}+R_{2} e^{a_{2} z}\right) \\
= & \frac{r}{2 \pi} \int_{0}^{2 \pi} \max \left\{\left|a_{1}\right| \cos \left(\theta+\theta_{1}\right),\left|a_{2}\right| \cos \left(\theta+\theta_{2}\right), 0\right\} d \theta+O(\log r) \\
= & \frac{r}{2 \pi} \int_{0}^{2 \pi} \max \left\{\left|a_{1}\right| \cos \left(\theta+\theta_{1}-\theta_{2}\right),\left|a_{2}\right| \cos \theta, 0\right\} d \theta+O(\log r) .
\end{aligned}
$$


Suppose that $\left|a_{1}\right|<\left|a_{2}\right|$. From (2.16) we have

$$
\begin{aligned}
T\left(r, R_{1} e^{a_{1} z}+R_{2} e^{a_{2} z}\right) & \geq \frac{r}{2 \pi} \int_{0}^{2 \pi} \max \left\{\left|a_{2}\right| \cos \theta, 0\right\} d \theta+O(\log r) \\
& =T\left(r, e^{a_{2} z}\right)+O(\log r) .
\end{aligned}
$$

From (2.15) and (2.17) we can obtain (2.14).

Suppose that $\left|a_{1}\right|=\left|a_{2}\right|$. Noting $a_{1} \neq a_{2}$, we may assume, without loss of generality, $0 \leq \theta_{2}<\theta_{1}<2 \pi$. If $\theta_{1}-\theta_{2} \leq \pi$, then $\frac{\pi}{2} \leq \frac{3 \pi}{2}-\theta_{1}+\theta_{2}<\frac{3 \pi}{2}$. From (2.16) we have

$$
\begin{aligned}
& T\left(r, e^{R_{1} a_{1} z}+R_{2} e^{a_{2} z}\right) \\
= & \frac{\left|a_{1}\right| r}{2 \pi} \int_{0}^{2 \pi} \max \left\{\cos \left(\theta+\theta_{1}-\theta_{2}\right), \cos \theta, 0\right\} d \theta+O(\log r) \\
\geq & \frac{\left|a_{1}\right| r}{2 \pi}\left\{\int_{0}^{\frac{\pi}{2}} \cos \theta d \theta+\int_{\frac{3 \pi}{2}-\theta_{1}+\theta_{2}}^{\frac{3 \pi}{2}} \cos \left(\theta+\theta_{1}-\theta_{2}\right) d \theta\right. \\
& \left.+\int_{\frac{3 \pi}{2}}^{2 \pi} \cos \theta d \theta\right\}+O(\log r) \\
= & \frac{\left|a_{1}\right| r}{2 \pi}\left(3-\cos \left(\theta_{1}-\theta_{2}\right)\right)+O(\log r) .
\end{aligned}
$$

From (2.15) and (2.18) we can obtain (2.14). If $\pi<\theta_{1}-\theta_{2}$, then $\frac{\pi}{2}<\frac{5 \pi}{2}-\theta_{1}+\theta_{2}<$ $\frac{3 \pi}{2}$. From (2.16) we have

$$
\begin{aligned}
& T\left(r, R_{1} e^{a_{1} z}+R_{2} e^{a_{2} z}\right) \\
= & \frac{\left|a_{1}\right| r}{2 \pi} \int_{0}^{2 \pi} \max \left\{\cos \left(\theta+\theta_{1}-\theta_{2}\right), \cos \theta, 0\right\} d \theta+O(\log r) \\
\geq & \frac{\left|a_{1}\right| r}{2 \pi}\left\{\int_{0}^{\frac{\pi}{2}} \cos \theta d \theta+\int_{\frac{\pi}{2}}^{\frac{5 \pi}{2}-\theta_{1}+\theta_{2}} \cos \left(\theta+\theta_{1}-\theta_{2}\right) d \theta\right. \\
& \left.+\int_{\frac{3 \pi}{2}}^{2 \pi} \cos \theta d \theta\right\}+O(\log r) \\
= & \frac{\left|a_{1}\right| r}{2 \pi}\left(3-\cos \left(\theta_{1}-\theta_{2}\right)\right)+O(\log r) .
\end{aligned}
$$

From (2.15) and (2.19) we can obtain (2.14).

This completes the proof of Lemma 2.8 . 


\section{Proof of Theorems}

Proof of Theorem 1.1. Suppose that $f$ is a polynomial, then from (1.3) we see that there exists a nonzero constant $c$ such that $e^{P(z)} \equiv c$. So $\sigma_{2}(f)=\gamma_{P}=0$, thus the conclusion (i) of Theorem 1.1 is valid. Next we suppose that $f$ is a transcendental entire function. We discuss the following two cases.

Case 1. Suppose that

$$
\sigma(f)=\infty
$$

From (3.1) and Lemma 2.1 we see that

$$
\sigma(f)=\limsup _{r \rightarrow \infty} \frac{\log \nu(r, f)}{\log r}=\infty,
$$

where $\nu(r, f)$ denotes the central-index of $f(z)$. If $P(z)$ is a constant, by (1.3) and Theorem 4.1 in [7] we deduce that all solutions of

$$
f^{(k)}+a_{k-1} f^{(k-1)}+\cdots+a_{2} f^{\prime \prime}+a_{1} f^{\prime}+\left(a_{0}-e^{P}\right) f=Q_{1}-Q_{2} e^{P}
$$

have finite order, this is a contradiction. Thus, $P(z)$ is a nonconstant polynomial. Let

$$
P(z)=p_{n} z^{n}+p_{n-1} z^{n-1}+\cdots+p_{1} z+p_{0},
$$

where $p_{n}(\neq 0), p_{n-1}, \cdots, p_{1}$ and $p_{0}$ are complex constants. It follows from (3.3) that

$$
\lim _{|z| \rightarrow+\infty} \frac{|P(z)|}{\left|p_{n} z^{n}\right|}=1
$$

From (3.4) we see that there exists a sufficiently large positive number $r_{0}$, such that

$$
\frac{|P(z)|}{\left|p_{n} z^{n}\right|}>\frac{1}{e} \quad\left(|z|>r_{0}\right) .
$$

From (1.3) and (3.5) we deduce

$$
\begin{aligned}
& n \log r+\log \left|p_{n}\right|-1 \\
= & \log \frac{\left|p_{n} z^{n}\right|}{e} \leq \log |P(z)|=\log \left|\log e^{P(z)}\right| \leq\left|\log \log e^{P(z)}\right| \\
= & \left|\log \log \frac{L[f]-Q_{1}}{f-Q_{2}}\right| \quad\left(|z|>r_{0}\right),
\end{aligned}
$$


On the other hand, since $f$ is a nonconstant entire function, thus

$$
M(r, f) \rightarrow+\infty
$$

as $r \rightarrow+\infty$, where $M(r, f)=\max _{|z|=r}|f(z)|$. Again let

$$
M(r, f)=\left|f\left(z_{r}\right)\right|,
$$

where $z_{r}=r e^{i \theta(r)}$, and $\theta(r) \in[0,2 \pi)$. From (3.8) and the Wiman-Valiron theory (see [7, Theorem 3.2]), we see that there exists a subset $E_{j} \subset(1, \infty)(1 \leq j \leq n)$ with finite logarithmic measure, i.e., $\int_{E_{j}} \frac{d t}{t}<\infty$, such that for some point $z_{r}=$ $r e^{i \theta(r)}(\theta(r) \in[0,2 \pi))$ satisfying $\left|z_{r}\right|=r \notin E_{j}$ and $M(r, f)=\left|f\left(z_{r}\right)\right|$, we have

$$
\frac{f^{(j)}\left(z_{r}\right)}{f\left(z_{r}\right)}=\left(\frac{\nu(r, f)}{z_{r}}\right)^{j}(1+o(1)) \quad(1 \leq j \leq n),
$$

as $r \rightarrow+\infty$. Noting that $f$ is a transcendental entire function, and $Q_{i}(i=1,2)$ are polynomials, from (3.1) and (3.8) we deduce

$$
\lim _{r \rightarrow \infty} \frac{\left|Q_{i}\left(z_{r}\right)\right|}{\left|f\left(z_{r}\right)\right|}=\lim _{r \rightarrow \infty} \frac{\left|Q_{i}\left(z_{r}\right)\right|}{M(r, f)}=0 \quad(i=1,2) .
$$

Since

$$
\frac{L[f]-Q_{1}}{f-Q_{2}}=\frac{\frac{L[f]}{f}-\frac{Q_{1}}{f}}{1-\frac{Q_{2}}{f}},
$$

from (1.2), (1.3), (3.2) and (3.6)-(3.11) we deduce

$$
n \log \left|z_{r}\right|+\log \left|p_{n}\right|-1 \leq\left|\log \log \left(\left(\frac{\nu(r, f)}{z_{r}}\right)^{k}(1+o(1))\right)\right|
$$

and

$$
\begin{aligned}
& \log \left(\left(\frac{\nu(r, f)}{z_{r}}\right)^{k}(1+o(1))\right) \\
= & k\left(\log \nu(r, f)-\log r e^{i \theta(r)}\right)+o(1) \\
= & k(\log \nu(r, f)-\log r-i \theta(r))+o(1) \\
= & k\left(1-\frac{\log r}{\log \nu(r, f)}-\frac{i \theta(r)}{\log \nu(r, f)}\right) \log \nu(r, f)+o(1),
\end{aligned}
$$


as $r \rightarrow+\infty$. Noting that $\theta(r) \in[0,2 \pi)$, from (3.2), (3.13) and Lemma 2.2 we deduce

$$
\begin{aligned}
& \limsup _{r \rightarrow \infty} \frac{\left|\log \log \left(\left(\frac{\nu(r, f)}{z_{r}}\right)^{k}(1+o(1))\right)\right|}{\log r} . \\
\leq & \limsup _{r \rightarrow \infty} \frac{\log \log \nu(r, f)}{\log r}+\limsup _{r \rightarrow \infty} \frac{\left|\log \left(1-\frac{\log r}{\log \nu(r, f)}-\frac{i \theta(r)}{\log \nu(r, f)}\right)\right|}{\log r} \\
& +\lim _{r \rightarrow \infty} \frac{\log 2}{\log r}+\lim _{r \rightarrow \infty} \frac{2 k_{1} \pi}{\log r} \\
= & \limsup _{r \rightarrow \infty} \frac{\log \log \nu(r, f)}{\log r}=\sigma_{2}(f),
\end{aligned}
$$

where $k_{1}$ is some nonnegative integer. Noting that $\left|z_{r}\right|=r$, from (3.12) and (3.14) we deduce

$$
n \leq \limsup _{r \rightarrow \infty} \frac{\log \log \nu(r, f)}{\log r}=\sigma_{2}(f) .
$$

From (3.3) we obtain

$$
\sigma\left(e^{P}\right)=\gamma_{P(z)}=n .
$$

From (3.15) and (3.16) we get

$$
\sigma\left(e^{P}\right) \leq \sigma_{2}(f) .
$$

If $\liminf _{r \rightarrow \infty}(\log \nu(r, f)) /(\log r)>1$, from (1.3), (3.2) and (3.9)-(3.11) we deduce

$$
\left(\frac{\nu(r, f)}{z_{r}}\right)^{k}(1+o(1))=e^{P\left(z_{r}\right)},
$$

as $r \rightarrow \infty$, and so it follows from (3.18) that

$$
\begin{aligned}
\limsup _{r \rightarrow \infty} \frac{\log \log \nu(r, f)}{\log r} & =\limsup _{r \rightarrow \infty} \frac{\log \log \left(\frac{\nu(r, f)}{2 r}\right)^{k}}{\log r} \\
& \leq \limsup _{r \rightarrow \infty} \frac{\log \log M\left(r, e^{P}\right)}{\log r} .
\end{aligned}
$$

From (3.19) and Lemma 2.2 we get

$$
\sigma_{2}(f) \leq \sigma\left(e^{P}\right) .
$$

From (3.16), (3.17) and (3.20) we get the conclusion (ii) of Theorem 1.1. 
Case 2. Suppose that

$$
\sigma(f)<\infty
$$

First, from (3.21) we can deduce

$$
\sigma_{2}(f)=0
$$

On the other hand, from (1.2), (1.3), (3.7)-(3.11), (3.21) and Lemma 2.1 we deduce

$$
\begin{aligned}
\left|P\left(z_{r}\right)\right|^{2}= & \left|\log \frac{L\left[f\left(z_{r}\right)\right]-Q_{1}\left(z_{r}\right)}{f\left(z_{r}\right)-Q_{2}\left(z_{r}\right)}\right|^{2} \\
= & \left(\log \left|\frac{L\left[f\left(z_{r}\right)\right]-Q_{1}\left(z_{r}\right)}{f\left(z_{r}\right)-Q_{2}\left(z_{r}\right)}\right|\right)^{2}+O(1) \\
\leq & \left(\operatorname { l o g } \left(\left(\frac{\nu(r, f)}{r}\right)^{k}+\left|a_{k-1}\right|\left(\frac{\nu(r, f)}{r}\right)^{k-1}+\cdots\right.\right. \\
& \left.\left.+\left|a_{2}\right|\left(\frac{\nu(r, f)}{r}\right)^{2}+\left|a_{1}\right|\left(\frac{\nu(r, f)}{r}\right)\right)\right)^{2}+O(1) \\
\leq & O\left((\log r)^{2}\right),
\end{aligned}
$$

as $r \rightarrow+\infty$. Since $P(z)$ is a polynomial, from (3.23) we deduce that $P(z)$ is a constant, and so $r_{P}=0$. From this and (3.22) we see that the conclusion of Theorem 1.1 is valid. If $\liminf _{r \rightarrow \infty}(\log \nu(r, f)) /(\log r) \leq 1$, then from $\mu(f)=\liminf _{r \rightarrow \infty}$ $(\log \nu(r, f)) /(\log r)$ which can be verified in the same manner as in the proof of Lemma 2.2 in [2], we have $\mu(f) \leq 1$. On the other hand, from (1.2) and (1.3) we get $T\left(r, e^{P}\right) \leq O(T(r, f)+\log T(r, f)+\log r)(r \notin E)$. From this and Lemma 1.1.1 in [7] we see that for sufficiently large positive number $r_{0}$, we have $T\left(r, e^{P}\right) \leq O(T(2 r, f)+\log T(2 r, f)+\log r+\log 2)\left(r \geq r_{0}\right)$. From this we get $1 \leq n=r_{P}=\sigma\left(e^{P}\right)=\mu\left(e^{P}\right) \leq \mu(f)$. Combining $\mu(f) \leq 1$, we get $\mu(f)=n=1$. Combining (1.3) and (3.3) we get $P(z)=p_{1} z+p_{0}$. If $a_{j}=0(0 \leq j \leq k-1)$, then $(1.3)$ can be rewritten by $f^{(k)}-Q_{1}=\left(f-Q_{2}\right) \cdot e^{p_{1} z+p_{0}}$. From this, Lemma 1.1.2 in [7] and in the same manner as in the proof of (3.16) we get $\mu_{2}(f)=r_{P}=1$. This contradicts $\mu(f)=1$. Thus $a_{0}, a_{1}, \cdots a_{k-2}$ and $a_{k-1}$ are not all equal to zero. From the above analysis we get (iii) of Theorem 1.1.

Theorem 1.1 is thus completely proved.

Proof of Theorem 1.3. Suppose that $f$ is a nonconstant polynomial. If $a_{0}=0$, it follows by (1.7) and (1.12) that $f$ is a polynomial of degree 2. Let

$$
f(z)=b_{2} z^{2}+b_{1} z+b_{0}
$$


where $b_{2}(\neq 0), b_{1}$ and $b_{0}$ are three finite complex numbers. From (1.7), (1.12) and (3.24) we deduce

$$
\frac{z-2 b_{2}}{\left(1-2 b_{2}\right) z-b_{1}}=e^{P},
$$

where $e^{p}$ is a constant. From this we deduce

$$
2 b_{2}-1 \neq 0
$$

and

$$
b_{1}=2 b_{2}\left(1-2 b_{2}\right) \text {. }
$$

Noting that

$$
f(z)-z=b_{2} z^{2}+\left(b_{1}-1\right) z+b_{0}
$$

and

$$
L[f]-z=f^{\prime}-z=\left(2 b_{2}-1\right) z+b_{1},
$$

from (3.27), (3.28) and the condition that $f(z)-z$ and $L[f](z)-z$ share 0 IM we can get

$$
\left(b_{1}-1\right)^{2}-4 b_{0} b_{2}=0
$$

and

$$
b_{1}+2 b_{2}=1 \text {. }
$$

From (3.25), (3.26) and (3.29) we get a contradiction. Thus,

$$
a_{0} \neq 0 .
$$

Then it follows from (1.7), (1.12) and (3.30) that $f$ is a polynomial of degree 1. Let

$$
f(z)=c_{1} z+c_{0},
$$

where $c_{1}(\neq 0)$ and $c_{0}$ are two finite complex numbers. From (1.7), (1.12) and (3.31) we deduce

$$
L^{\prime}[f](z)-z=a_{0} c_{1}-z
$$

and

$$
\frac{a_{0} c_{1}-z}{\left(a_{0} c_{1}-1\right) z+a_{0} c_{0}+c_{1}}=e^{P},
$$


which implies that $1-a_{0} c_{1} \neq 0$,

$$
e^{P} \equiv \frac{1}{1-a_{0} c_{1}}
$$

and

$$
\frac{a_{0} c_{0}+c_{1}}{1-a_{0} c_{1}}=a_{0} c_{1} .
$$

Noting that $f(z)-z$ and $L^{\prime}[f](z)-z$ share $0 \mathrm{IM}$, from (3.31) and (3.32) we deduce

$$
c_{1}-1 \neq 0
$$

and

$$
c_{0}=a_{0} c_{1}\left(1-c_{1}\right) .
$$

Substituting (3.36) into (3.34) we deduce

$$
a_{0}^{2}-a_{0}+1=0 .
$$

From (3.31), (3.33) and (3.35)-(3.37) we can get the conclusion (i) of Theorem 1.3.

Next we suppose that $f$ is a transcendental entire function. First, by Milloux's inequality (see [5, Theorem 3.2]) we have

$$
T(r, f)<N\left(r, \frac{1}{f(z)-z}\right)+\bar{N}\left(r, \frac{1}{L^{\prime}[f](z)-z}\right)+S(r, f) .
$$

Let $z_{0}$ be a zero of $f(z)-z$ with multiplicity $\geq 2$. Then $f\left(z_{0}\right)=z_{0}$ and $f^{\prime}\left(z_{0}\right)=1$. Since $f$ and $L[f]$ share $z \mathrm{IM}$, we have $L[f]\left(z_{0}\right)=z_{0}$. Thus $1+a_{0} z_{0}=z_{0}$, and hence $f(z)-z$ has at most one zero with multiplicity $\geq 2$. From this we obtain

$$
\begin{aligned}
N\left(r, \frac{1}{f(z)-z}\right) & =\bar{N}\left(r, \frac{1}{f(z)-z}\right)+O(\log r) \\
& =\bar{N}\left(r, \frac{1}{L[f](z)-z}\right)+O(\log r) .
\end{aligned}
$$

From (1.12) we have

$$
\bar{N}\left(r, \frac{1}{L^{\prime}[f](z)-z}\right)=\bar{N}\left(r, \frac{1}{L[f](z)-z}\right) .
$$

From (3.38), (3.40) and (3.41) we obtain

$$
\begin{aligned}
T(r, f) & \leq 2 \bar{N}\left(r, \frac{1}{L[f](z)-z}\right)+S(r, f) \\
& \leq 2 T(r, L[f])+S(r, f) \leq 2 T(r, f)+S(r, f) .
\end{aligned}
$$


From (3.42) we deduce

$$
\sigma_{2}(f)=\sigma_{2}(L[f]) .
$$

From (3.43) and the condition that $\sigma_{2}(f)$ is not a positive integer we know that $\sigma_{2}(L[f])$ is not a positive integer, and so it follows from (1.12) and Theorem 1.2 that there exists a finite nonzero complex number $d$ such that

$$
\frac{L^{\prime}[f]-z}{L[f]-z} \equiv d
$$

We discuss the following three cases.

Case 1. Suppose that $a_{0}=0$. Then it follows from (1.7) that (3.44) can be rewritten by

$$
f^{\prime \prime}-d f^{\prime}=(1-d) z
$$

From (3.45) we deduce

$$
f=d_{1} e^{d z}+\frac{d-1}{2 d} z^{2}+\frac{d-1}{d^{2}} z+d_{2},
$$

where $d_{1}(\neq 0)$ and $d_{2}$ are constants. Thus,

$$
\begin{gathered}
f-z=d_{1} e^{d z}+\frac{d-1}{2 d} z^{2}+\frac{d-1-d^{2}}{d^{2}} z+d_{2}, \\
L[f]-z=d_{1} d e^{d z}-\frac{1}{d} z+\frac{d-1}{d^{2}} .
\end{gathered}
$$

Assume that $d \neq 1$. Since $f(z)-z$ and $L[f]-z$ share 0 IM, by Lemma 2.7, (3.47) and (3.48) we get a contradiction. Thus $d=1$, and so it follows from (3.47) and (3.48) that $f-z=d_{1} e^{z}-z+d_{2}$ and $L[f]-z=d_{1} e^{z}-z$. Combining the condition that $f-z$ and $L[f]-z$ share 0 IM we deduce $d_{2}=0$, and so it follows that $f=d_{1} e^{z}$, which reveals the conclusion (ii) of Theorem 1.3.

Case 2. Suppose that $a_{0} \neq 0$ and $a_{0}=-d$. Then it follows from (1.7) and (3.44) that

$$
f^{\prime \prime}-2 d f^{\prime}+d^{2} f=(1-d) z .
$$

From (3.49) we deduce

$$
f(z)=\left(d_{3} z+d_{4}\right) e^{d z}+\frac{1-d}{d^{2}} z+\frac{2(1-d)}{d^{3}},
$$


where $d_{3}$ and $d_{4}$ are constants satisfying $d_{3} z+d_{4} \not \equiv 0$. Thus,

$$
\begin{gathered}
f(z)-z=\left(d_{3} z+d_{4}\right) e^{d z}+\frac{1-d-d^{2}}{d^{2}} z+\frac{2(1-d)}{d^{3}}, \\
L[f]-z=d_{3} e^{d z}-\frac{1}{d} z+\frac{d-1}{d^{2}} .
\end{gathered}
$$

By Lemma 2.7, (3.50) and (3.51) we get a contradiction.

Case 3. Suppose that $a_{0} \neq 0$ and $a_{0} \neq-d$. Then it follows from (1.7) and (3.44) that

$$
f^{\prime \prime}+\left(a_{0}-d\right) f^{\prime}-a_{0} d f=(1-d) z .
$$

From (3.52) we deduce

$$
f=d_{5} e^{-a_{0} z}+d_{6} e^{d z}+\frac{d-1}{a_{0} d} z+\frac{\left(a_{0}-d\right)(d-1)}{a_{0}^{2} d^{2}},
$$

where $d_{5}$ and $d_{6}$ are constants satisfying $d_{5} e^{-a_{0} z}+d_{6} e^{d z} \not \equiv 0$. Thus,

$$
\begin{aligned}
& f-z=d_{5} e^{-a_{0} z}+d_{6} e^{d z}+P_{1}(z), \\
& L[f]-z=d_{6}\left(d+a_{0}\right) e^{d z}+P_{2}(z),
\end{aligned}
$$

where

$$
\begin{aligned}
& P_{1}(z)=\frac{d-1-a_{0} d}{a_{0} d} z+\frac{\left(a_{0}-d\right)(d-1)}{a_{0}^{2} d^{2}}, \\
& P_{2}(z)=-\frac{1}{d} z+\frac{d-1}{d^{2}} .
\end{aligned}
$$

If $d_{5}=0$, then $d_{6} \neq 0$. By Lemma 2.7, (3.53) and (3.54) we get a contradiction. If $d_{6}=0$, then $d_{5} \neq 0$. From (3.53) and (3.54) we obtain a contradiction. Next, we suppose that $d_{5} \neq 0$ and $d_{6} \neq 0$.

Let $z_{0}$ be a zero of $L[f]-z$. From (3.54) we obtain

$$
d_{6}\left(d+a_{0}\right) e^{d z_{0}}+P_{2}\left(z_{0}\right)=0 .
$$

Since $f-z$ and $L[f]-z$ share 0 IM, from (3.53) we deduce

$$
d_{5} e^{-a_{0} z_{0}}+d_{6} e^{d z_{0}}+P_{1}\left(z_{0}\right)=0 .
$$

From (3.55) and (3.56) we have

$$
d_{5}\left(d+a_{0}\right) e^{-a_{0} z_{0}}+\left(d+a_{0}\right) P_{1}\left(z_{0}\right)-P_{2}\left(z_{0}\right)=0 .
$$


Noting that $z_{0}$ is a zero of $L[f]-z$, from (3.55) and (3.57) we obtain

$$
\begin{aligned}
& \bar{N}\left(r, \frac{1}{d_{6}\left(d+a_{0}\right) e^{d z}+P_{2}(z)}\right) \\
\leq & \bar{N}\left(r, \frac{1}{d_{5}\left(d+a_{0}\right) e^{-a_{0} z}+\left(d+a_{0}\right) P_{1}(z)-P_{2}(z)}\right) .
\end{aligned}
$$

It is easy to see that

$$
\begin{aligned}
& T\left(r, e^{d z}\right)=\bar{N}\left(r, \frac{1}{d_{6}\left(d+a_{0}\right) e^{d z}+P_{2}(z)}\right)+O(\log r), \\
& T\left(r, e^{-a_{0} z}\right) \\
= & \bar{N}\left(r, \frac{1}{d_{5}\left(d+a_{0}\right) e^{-a_{0} z}+\left(d+a_{0}\right) P_{1}(z)-P_{2}(z)}\right)+O(\log r) .
\end{aligned}
$$

From (3.58)-(3.60) we deduce

$$
T\left(r, e^{d z}\right) \leq T\left(r, e^{-a_{0} z}\right)+O(\log r) .
$$

Since

$$
T\left(r, e^{d z}\right)=\frac{|d| r}{\pi} \quad \text { and } \quad T\left(r, e^{-a_{0} z}\right)=\frac{\left|a_{0}\right| r}{\pi},
$$

from (3.61) we get $|d| \leq\left|a_{0}\right|$. Noting that $d \neq-a_{0}$, by Lemma 2.8, (3.53) and (3.54) we know that there exists a constant $A(>1)$ such that

$$
A T(r, L[f]) \leq T(r, f)+O(\log r) .
$$

On the other hand, from (3.54) we have

$$
T(r, L[f])=\bar{N}\left(r, \frac{1}{L[f]-z}\right)+O(\log r) .
$$

By Lemma 2.7 and the condition that $f-z$ and $L[f]-z$ share 0 IM, we deduce $P_{1}(z) \not \equiv 0$. Combining (3.53), (3.54) and Lemma 2.5 we deduce

$$
T(r, f)=N\left(r, \frac{1}{f-z}\right)+O(\log r) .
$$

Again from (3.39) and (3.64) we obtain

$$
T(r, f)=\bar{N}\left(r, \frac{1}{f-z}\right)+O(\log r) .
$$

Since $f-z$ and $L[f]-z$ share 0 IM, we have

$$
\bar{N}\left(r, \frac{1}{f-z}\right)=\bar{N}\left(r, \frac{1}{L[f]-z}\right) .
$$


From (3.63), (3.65) and (3.66) we obtain

$$
T(r, L[f])=T(r, f)+O(\log r) .
$$

Noting that $f$ is a transcendental entire function, from (3.62) and (3.67) we get a contradiction.

Theorem 1.3 is thus completely proved.

\section{ACKNOWLEDGMENT}

The authors would like to thank the referee for valuable suggestions concerning this paper.

\section{REFERENCES}

1. R. Brück, On entire functions which share one value $\mathrm{CM}$ with their first derivative, Results in Math., 30 (1996), 21-24.

2. Z. X. Chen and C. C. Yang, Some further results on the zeros and growths of entire solutions of second order linear differential equation, Kodai Math J., 22 (1999), 273-285.

3. Z. X. Chen, The growth of solutions of $f^{\prime \prime}+e^{-z} f^{\prime}+Q(z) f=0$ Science in China (A), 31 (2001), 775-784.

4. G. G. Gundersen and L. Z. Yang, Entire functions that share one value with one or two of their derivatives, J. Math. Anal. Appl., 223 (1998), 88-95.

5. W. K. Hayman, Meromorphic Functions, Clarendon Press, Oxford, 1964.

6. G. Jank and L. Volkmann, Einführung in die Theorie der ganzen und meromorphen Funktionen mit Anwendungen auf Differentialgleichungen, Birkhäuser, Basel-Boston, 1985.

7. I. Laine, Nevanlinna Theory and Complex differential Equations, Walter de Gruyter, Berlin, 1993.

8. L. Rubel and C. C. Yang, Values shared by an entire function and its derivative, in: Complex Analysis, Kentucky 1976, (Proc. Conf.), Lecture Notes in Mathematics, Vol 599, Springer-Verlag, Berlin, 1977, pp. 101-103.

9. L. Yang, Value distribution theory, Berlin Heidelberg: Springer-Verlag, Beijing: Science Press, 1993.

10. L. Z. Yang, Solution of a differential equation and its applications, Kodai Math J., 22 (1999), 458-464. 
11. C. C. Yang and H. X. Yi, Uniqueness Theory of Meromorphic Functions, Mathematics and Its Applications Vol. 557, Kluwer Academic Publishers, Dordrecht, 2003.

\author{
Xiao-Min Li \\ Department of Mathematics, \\ Ocean University of China, \\ Qingdao, Shandong 266071, \\ P. R. China \\ E-mail:xmli01267@gmail.com \\ Hong-Xun Yi \\ Department of Mathematics, \\ Shandong University, \\ Jinan, Shandong 250100, \\ P. R. China \\ E-mail: hxyi@sdu.edu.cn
}

